\title{
Inhaltsverzeichnis
}

Seite

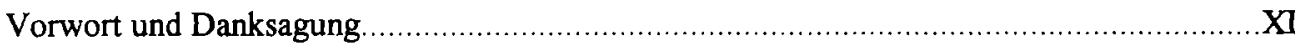

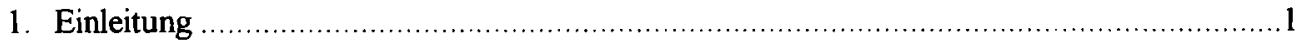

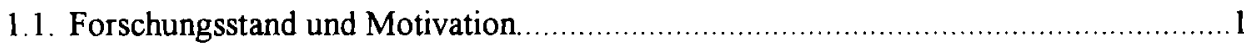

1.2. Anforderungen an phonetische Modelle der Sprachproduktion und ihre

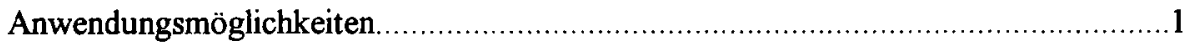

1.3. Zum Aufbau eines phonetischen Modells der Sprachproduktion.............................2

1.4. Relationen zwischen Artikulation, Akustik und Perzeption aus der Sicht der Sprachproduktion..................................................................................4

1.5. Vollständige und konkrete phonetische Modelle der Sprachproduktion.....................4

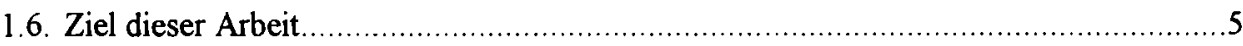

\section{THEORETISCHER TEIL}

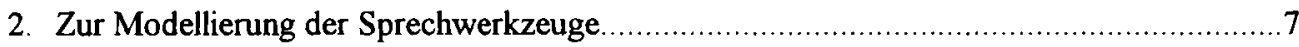

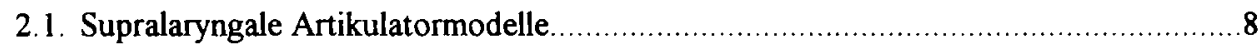

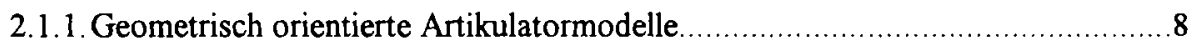

2.1.2. Effektorisch orientierte Artikulatormodelle ........................................ 10

2.1.3. Statistisch orientierte Artikulatormodelle ............................................. 12

2.1.4. Physiologisch orientierte Artikulatormodelle ............................................ 12

2.1.5. Akustisch orientierte Artikulatormodelle ............................................. 14

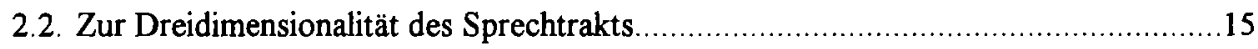

2.2.1. Von der Mediosagittalkontur zur Areafunktion.................................... 15

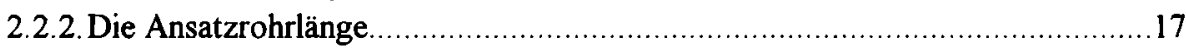

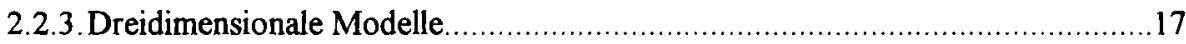

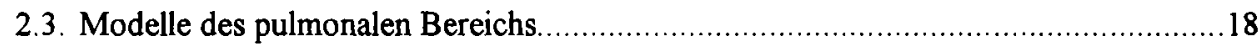

2.3.1. Das Modell konstanten Drucks............................................................. 19

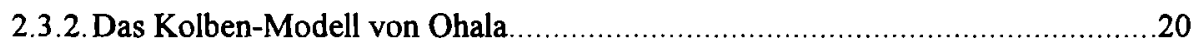

2.3.3. Das physiologische Modell von Rothenberg.........................................21

2.3.4. Akustische Modelle des subglottalen Bereichs............................................22

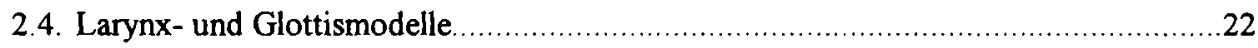

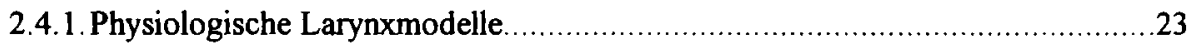

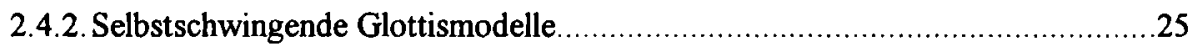

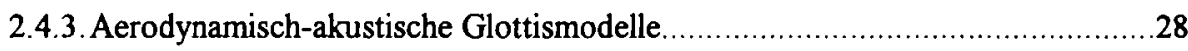

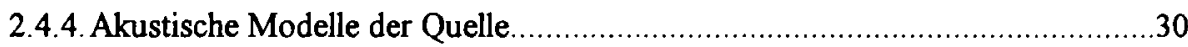

2.5. Akustische und aerodynamische Modellierung des Sprechtraktes............................ 31

2.5.1. Akustische Modelle des Sprechtraktes.................................................... 31

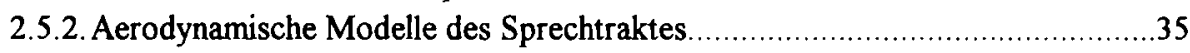

2.5.3. Die Verluste im Sprechtrakt.............................................................. 
2.5.4. Die aerodynamisch-akustischen Mechanismen an der glottalen Konstriktion... 41

2.2.5. Die Schallabstrahlung an Mund und Nase ..........................................42

2.5.6. Der Nasaltrakt und die Nebenhohlräume des Sprechtraktes.........................43

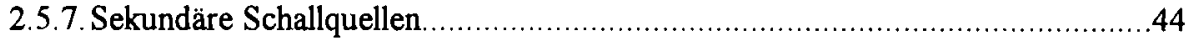

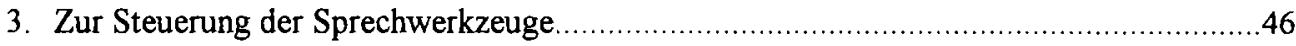

3.1. Das Problem der phonetischen Variabilität................................................46

3.1.1. Artikulatorische, akustische und perzeptive Variabilität ..........................47

3.1.2. Artikulatorische Invarianz: "Eisberge" und Gesten...................................48

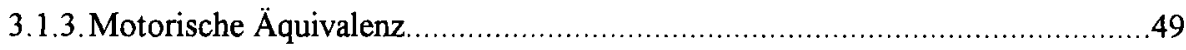

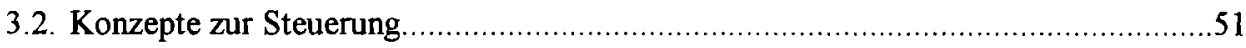

3.2.1. Artikulatorische und akustische Steuerung .........................................51

3.2.2. Kinematische und dynamische Modelle.................................................53

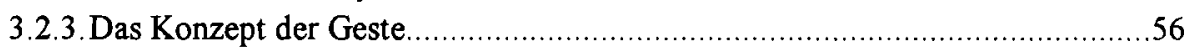

3.3. Zur Analyse artikulatorischer Daten in Hinblick auf Modelle der Steuerung.............60

3.3.1. Artikulatorische Meßmethoden........................................................60

3.3.2. Die kinematischen Parameter der Sprechbewegungen.................................62

3.3.3. Die dynamischen Parameter der Sprechbewegungen..................................63

3.4. Die Modellierung prosodisch bedingter Variabilität........................................65

3.4.1. Hypo- und Hyperartikulation...........................................................66

3.4.2. Artikulatorisch-gestische Korrelate zu Akzentuierung, Sprechtempo-

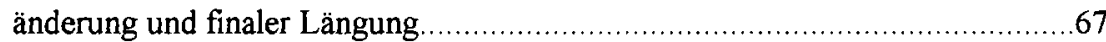

3.4.3. Reduktionsphänomene: Zur Variabilität diskreter segmentaler Formen in

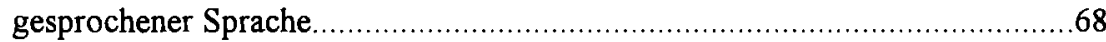

3.5. Segmentale und prosodische Aspekte der laryngalen Artikulation.........................69

3.5.1. Register und Phonationstypen.........................................................69

3.5.2. Laryngale Artikulation zur Lautrealisation....................................... 71

3.5.3. Artikulatorische Modelle zur Intonation............................................. 72

\section{EXPERIMENTELLER TEIL}

4. Das implementierte phonetische Modell der Sprachproduktion PHOPRO:

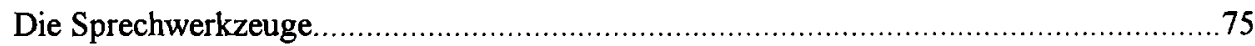

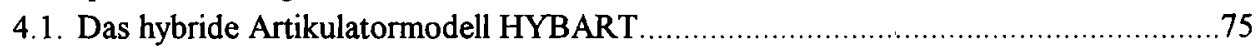

4.1.1. Artikulatoren, Steuerparameter und mediosagittale Konturen.........................76

4.1.2. Akustische Basierung der Extremalkonturen des Zungenrückens..................79

4.1.3. Beschreibung der Ansatzrohrkonstriktionen mittels der artikulatorischen

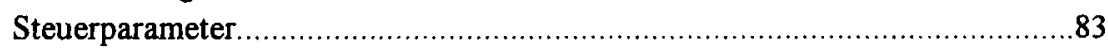

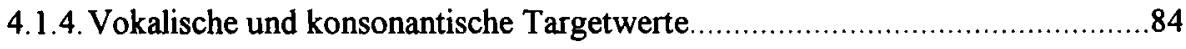

4.1.5. Die Berechnung der Areafunktion........................................................ 85

4.1.6. Eigenschaften des Modells I: Der Zusammenhang zwischen Steuerparametereinheiten, mediosagittaler Distanz und Querschnittsfläche des Ansatzrohres...87

4.1.7. Eigenschaften des Modells II: Zur Auswahl der artikulatorischen

Steuerparameter und zur Generierung von Zungenrückenkonstriktionen. 
4.2. Das Modell des pulmonalen Bereiches und der Trachea PULTRA ........................88

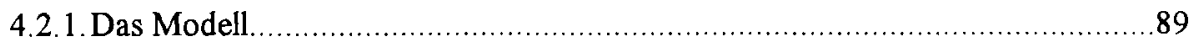

4.2.2. Eigenschaften des Modells: Pulmonaler und subglottaler Druck und der

Effekt der Trägheit der Luftsäule auf den Volumenstromimpuls.......................90

4.2.3.Zur Abschätzung der Konstanten des Modells des pulmonalen Bereichs.........91

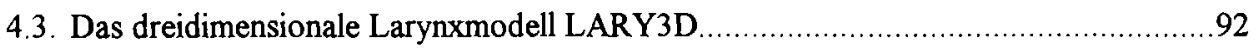

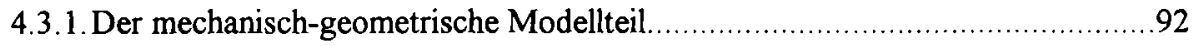

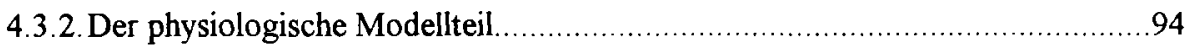

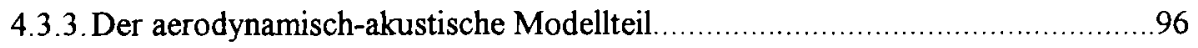

4.3.4. Eigenschaften des Modells I: Die Steuerparameter und das

Schwingungsverhalten der Stimmlippen.....................................................97

4.3.5. Eigenschaften des Modells II: Zur Erweitenung des Zwei-Masssen-Modells...100

4.4. Das aerodynamisch-akustische Modell des Sprechtraktes AERAKU......................101

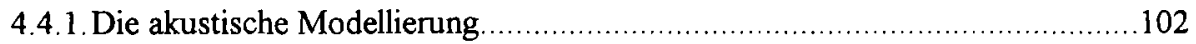

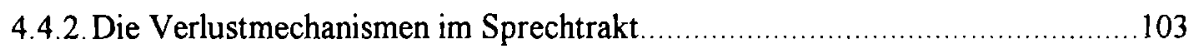

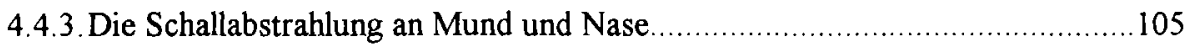

4.4.4. Die glottalen Verluste und die Kopplung zum pulmonalen Bereich.................107

4.4.5. Die Modellierung der sekundären Schallquellen..........................................109

4.4.6. Eigenschaften des Modells I: Der Volumenstrom als Funktion der

Querschnittsfläche der laryngalen und supralaryngalen Konstriktionen...........110

4.4.7. Eigenschaften des Modells II: Vergleich mit anderen Modellen......................111

5. Das implementierte phonetische Modell der Sprachproduktion PHOPRO:

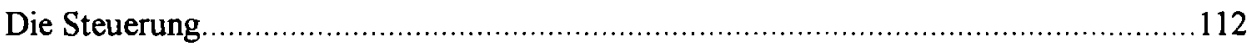

5.1. Das segmentale Modell SEGMOD

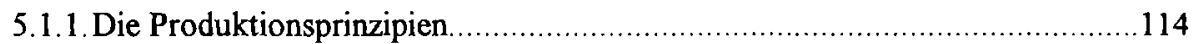

5.1.2. Das kinematische Modell zur Realisierung der artikulatorischen

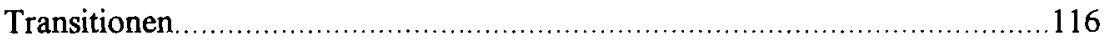

5.1.3. Zur quantitativen Spezifikation der Produktionsmerkmale ............................117

5.1.4. Beschreibung der Produktion unterschiedlicher Lauttypen ............................118

5.1.5. Distinktive Merkmale und Produktionsmerkmale ........................................ 123

5.1.6. Zur Produktion eines konstanten Konstriktionsgrades: Der Effekt der

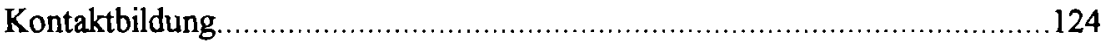

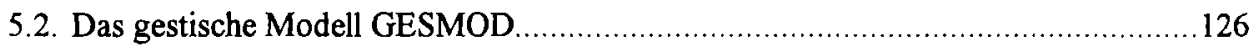

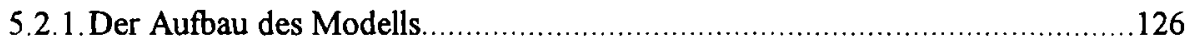

5.2.2. Gestentypen und der Aufbau ganzer Äußerungen.....................................127

5.2.3. Das dynamische Modell der Geste und der Parameter Eigenperiodendauer....131

5.2.4. Das Abschneiden gestischer Steuerparameterzeitfunktionen und das Kon-

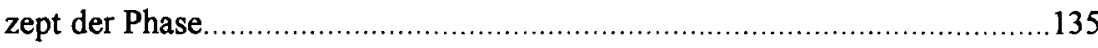

5.2.5. Die Generierung der gestischen Partitur: Die intergestischen Steuerpara-

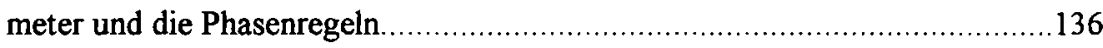

5.2.6. Zur quantitativen Abschätzung der gestischen Parameter ............................145

5.2.7. Gesteninventar und gestische Unterspezifikation........................................ 148 
5.2.8. Prioritätsregeln bei zeitlich überlappenden Gesten ......................................149

5.3. Vergleich des gestischen und des segmentalen Ansatzes.....................................151

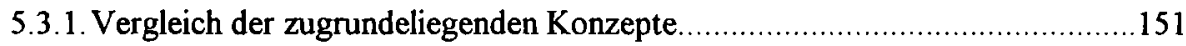

5.3.2. Vergleich der generierten Steuerparameterzeitfunktionen .............................152

6. Experimentelle Untersuchungen I: Perzeptive Evaluierung und akustische Analysen.......153

6.1. Perzeptive Analyse des segmentalen Modells SEGMOD ......................................153

6.1.1.Zum Begriff der Sprachqualität und die Testmethoden.................................153

6.1.2. Die globale Evaluierung des Synthesesystems.............................................153

6.1.3. Diskussion der Ergebnisse und Ausblick.....................................................155

6.2. Perzeptive Evaluierung von reduzierten Formen des Deutschen im gestischen

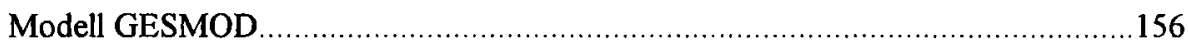

6.2.1. Qualitative perzeptive Analysen einer segmentalen Änderung aufgrund einer gestischen Verschiebung innerhalb einer Äußerung...............................156

6.2.2. Quantitative perzeptive Analyse zur regressiven und progressiven

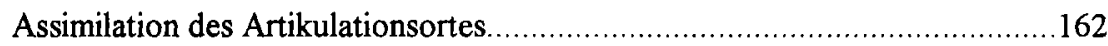

6.2.3. Qualitative perzeptive Analysen mehrerer segmentaler Änderungen aufgrund von gestischen Verschiebungen und aufgrund von gestischer Reorganisation am Beispiel "mit dem".

6.2.4. Quantitative perzeptive Analyse segmentaler Änderungen am

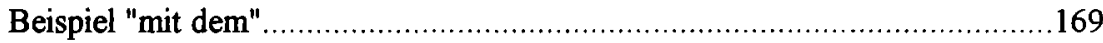

6.2.5. Diskussion der Ergebnisse der Perzeptionstests zur Reduktion......................170

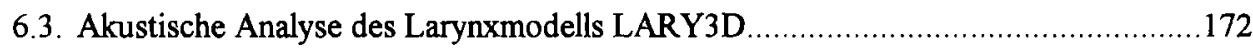

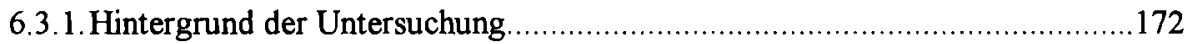

6.3.2. Grundfrequenz und Intensität als Funktion von Stimmlippen-

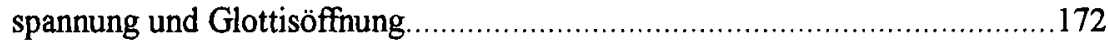

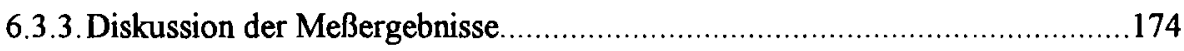

7. Experimentelle Untersuchungen II: Messung von Artikulatorbewegungen.....................175

7.1. Vorbemerkungen zur Abschätzung gestischer Parameter anhand artikula-

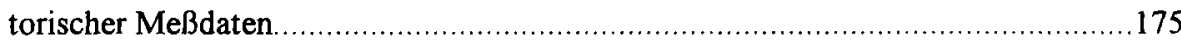

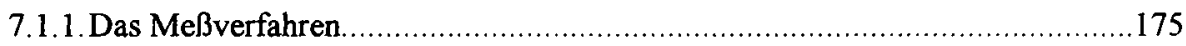

7.1.2. Erste gestische Analysen artikulatorischer Daten.....................................177

7.2. Ein erweitertes gestisches Modell und das Meßverfahren zur Abschätzung

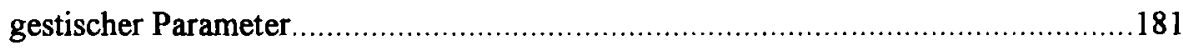

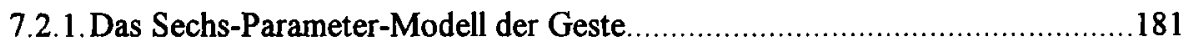

7.2.2. Die Aufgabe des Anpassungsverfahrens und das Minimierungskriterium........183

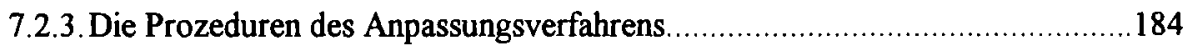

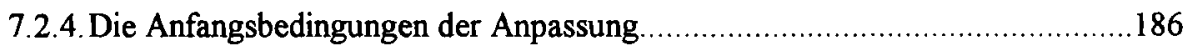

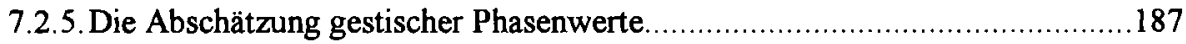

7.3. Evaluierung des Anpassungsverfahrens zur Messung gestischer Parameter.............188

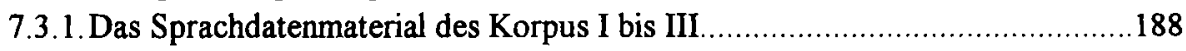


7.3.2. Die Anpassungsgüte der Zeitfunktionen des Sechs-Parameter-Modells und die Genauigkeit der Parameterschätzung

7.3.3. Die Abschätzung der Targetposition.

7.3.4. Die Abschätzung der zeitlichen Lage des Anklingintervalls und der Eigenperiodendauer

7.4. Die Änderung gestischer Parameter aufgrund prosodischer Faktoren

7.4.1. Das Sprachdatenmaterial des Korpus IV bis VII und die Analyseparameter

7.4.2. Die Änderung gestischer Parameter durch den prosodischen Faktor Akzentuierung.

7.4.3. Die Änderung gestischer Parameter durch die prosodischen Faktoren Akzentuierung und Sprechgeschwindigkeit .200

7.4.4. Die Änderung gestischer Parameter durch den prosodischen Faktor Finalität

7.4.5.Zur Gewichtung der Änderungen der gestischen Parameter durch die unterschiedlichen prosodischen Faktoren

7.4.6. Zur Interpretation der Änderung der gestischen Parameter aufgrund prosodischer Faktoren aus der Sicht der Steuerung

\section{EIN GESTISCHER ANSATZ ZUR PROSODISCHEN STEUERUNG}

8. Prosodische Aspekte der Steuerung: Modellierung artikulatorischer Phänomene...........223

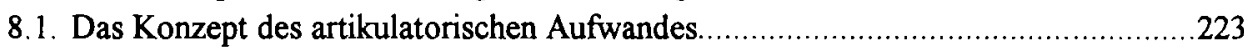

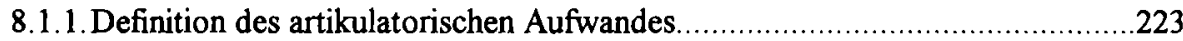

8.1.2. Quantifizierung des artikulatorischen Aufwandes......................................224

8.1.3. Abschätzung des artikulatorischen Aufwandes anhand von Meßdaten und seine Korrelation mit der gestischen Maximalgeschwindigkeit .226

8.2. Die Änderung gestischer Steuerparameter aufgrund der prosodischen Faktoren Akzentuierung, Sprechgeschwindigkeit und Finalität

8.2.1. Das gestisch-prosodische Modell der Akzentuierung, der Sprechgeschwindigkeit und der Finalität

8.2.2. Die Variierung der Targetposition und das Problem der segmentalen Invarianz

8.2.3. Diskussion des gestisch-prosodischen Modells anhand von Literaturdaten.... 235

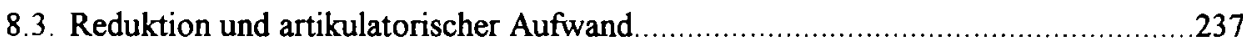

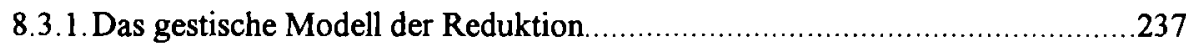

8.3.2. Die gestischen Verschiebungsprozesse .................................................239

8.3.3. Der Austausch von Gesten: Assimilation des Artikulationsortes und Glottalisierung im Deutschen.

9. Prosodische Aspekte der Steuerung: Modellierung phonatorischer Phänomene.............242

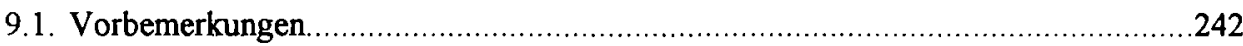

9.1.1. Akzentuierung und Intonation als prosodische Kategorien.......................242 
9.1.2.Zum Fehlen artikulatorischer Produktionsmodelle zur Akzentuierung und Intonation.

9.1.3.Zugrundeliegende pulmonale und laryngale Steuerparameter..

9.2. Zwei Ansätze zur artikulatorischen Modellierung prosodisch-phonatorischer Phänomene.

9.2.1. Die PS/CT-Steuerung ohne pulmonales Modell.

9.2.2. Die GA/CT-Steuerung mit pulmonalem Modell.

9.2.3. Die Kopplung zur supraglottalen Komponente: Ein prosodisches Gesamtmodell der laryngalen und supralaryngalen Artikulation für die Akzentuierung...249

9.3. Diskussion der GA/CT-Steuerung.........................................................250

9.3.1. Grundfrequenz und Intensität als Funktion artikulatorischer Variablen..........250

9.3.2.Zur Steuerung des subglottalen Drucks...............................................251

9.3.3. Akustische Konsequenzen glottaler Abduktion und Adduktion....................252

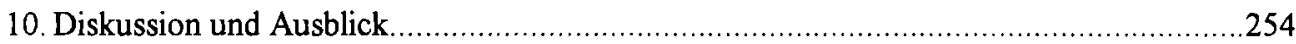

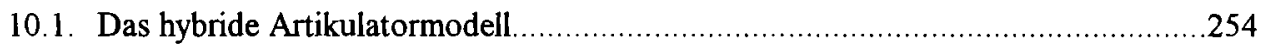

10.2. Das Modell des pulmonalen Bereichs und der Trachea, das Larynxmodell und das aerodynamisch-akustische Modell des Sprechtrakts.............................255

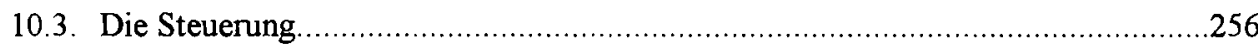

10.4. Theoretische Aspekte des gestischen Ansatzes..............................................257

10.5. Zur akustischen Rigidität und zur Evaluierung der Qualität des

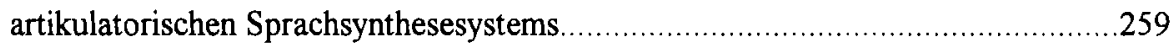

10.6. Gestische Verschiebungs- und Reorganisationsprozesse................................260

10.7. Zur Ermittlung gestischer Parameter und das quantitative Modell der Geste.........262

10.8. Zur gestisch-prosodischen Analyse artikulatorischer Daten .............................264

10.9. Zur zeitlichen Steuerung eines artikulatorisch-gestischen Produktionsmodells..... 266

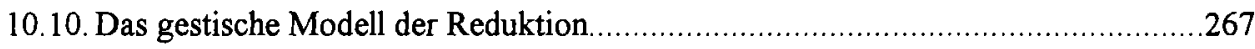

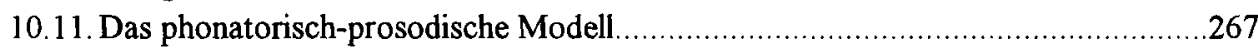

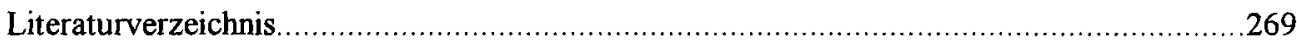

ANHANG

Al. Programmaufbau und Dateistruktur des Produktionsmodells................................286

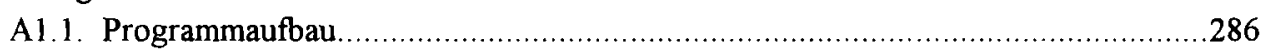

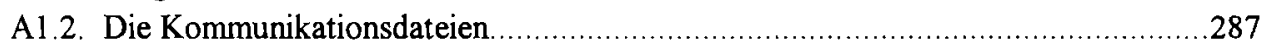

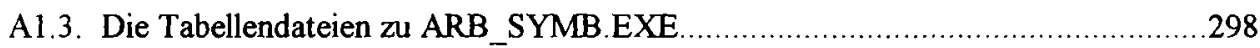

A2. Zur perzeptiven Analyse des segmentalen Modells...............................................306

A2.1. Textvorlagen zur perzeptiven Analyse des segmentalen Modells....................306

A2.2. Tabelle zur Fehlerhäufigkeit von Wörtern in der perzeptiven Analyse des segmentalen Modells. 